Аксенюшкина E.B.

\title{
Оптимальная финансовая политика фирмы
}

Байкальский государственнылй университет (Россия, Иркутск)

doi:10.18411/spc-8-12-2017-02

idsp: 000001:spc-8-12-2017-02

В статье формулируется и решается задача о формировании кредитной политики фирмы и таком распределении прибыли на инвестиции (процентный рост) и потребление (дивиденды), чтобы обеспечить максимальный объем потребления за некоторый период планирования. Рассматривается ситуация, когда некоторая фирма (например, банк) осуществляет свою деятельность на кредитно-финансовом рынке. Поясним механизм этой деятельности. Фирма имеет некоторый собственный капитал (собственные средства), который складывается из средств владельцев фирмы, накопленных за период существования. Далее фирма может привлекать средства клиентов, например, в виде вкладов, по некоторой процентной ставке, неся при этом расходы по выплате процентов за использование этих средств. Итак, имея в своем распоряжении собственные и привлеченные средства, фирма может разместить их (к примеру, в качестве выдаваемых кредитов) по существующей процентной ставке, получая, тем самым, некоторый доход.

Описанная ситуация формализуется в рамках билинейной задачи оптимального управления. В таких задачах, вообе говоря, принцип максимума является только необходимым условием оптимальности и выделяет экстремальные управления, которые подозрительны на оптимальность. С учетом специфики задачи показывается, что управления, удовлетворяющие принципу максимума, являются сильно экстремальными, что согласно известным достаточным условиям обеспечивает их оптимальность. В результате рассматриваемая билинейная задача полностью решается на основе принципа максимума.

Поставим задачу оптимального планирования со следующими переменными:

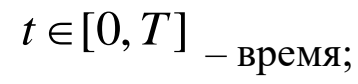

$x(t)$ - собственные средства фирмы в момент времени $t$, которые могут быть использованы на инвестиции и потребление;

$y(t)$ - привлеченные средства (например, кредит) в момент времени $t$;

$r_{1} \in(0,1]$ - процентная ставка по инвестициям;

$r_{2} \in(0,1]_{-}$процентная ставка по привлечению средств;

$v(t) \in[0,1]$ - доля собственных средств, идущая на инвестиции в момент времени $t$;

$u(t) \in[0,1]$ - отношение привлеченных средств к собственным в момент $t$ (объем привлеченных средств не превосходит собственные)

$$
u(t)=\frac{y(t)}{x(t)} .
$$

Тогда величину $x(t)$ можно представить в виде (кредит $y(t)$ расходуется только на инвестиции после уплаты процентов)

$$
x(t)=x(0)+r_{1} \int_{0}^{t} x(\tau) v(\tau) d \tau+r_{1}\left(\int_{0}^{t} y(\tau) d \tau-r_{2} \int_{0}^{t} y(\tau) d \tau\right)=
$$




$$
=x(0)+r_{1} \int_{0}^{t} x(\tau) v(\tau) d \tau+r_{1}\left(1-r_{2}\right) \int_{0}^{t} x(\tau) u(\tau) d \tau
$$

Обозначим $r=r_{1}, \quad c=1-r_{2}, \quad 0<c<1$. Следовательно, собственные средства фирмы можно записать в виде

$$
x(t)=x(0)+r \int_{0}^{t} x(\tau)[v(\tau)+c u(\tau)] d \tau .
$$

В результате дифференцирования получаем фазовое уравнение

$$
\dot{x}=r(c u+v) x
$$

с начальным условием $x(0)=x_{0}$.

Сформируем целевой функционал, подлежащий максимизации:

$$
\Phi(u, v)=\int_{0}^{T}(1-v(t)) x(t) d t-\int_{0}^{T} u(t) x(t) d t .
$$

По содержательному смыслу это разность между собственными средствами фирмы, направленными на потребление, и привлеченными средствами за период $[0, T]$ $\rho>0$

Примем предположение об обесценивании со временем капитала фирмы. Пусть обесценивания средств, запланированных на потребление, целевой функционал примет окончательный вид

$$
\Phi(u, v)=\int_{0}^{T} e^{-\rho t}(1-u(t)-v(t)) x(t) d t
$$

В результате приходим к задаче оптимального управления на максимум потребления

$$
\begin{gathered}
\Phi(u, v)=\int_{0}^{T} e^{-\rho t}(1-u-v) x d t \rightarrow \max , \\
\dot{x}=r(c u+v) x, \quad x(0)=x_{0}, \\
u(t) \in[0,1], \quad v(t) \in[0,1], \\
x_{0}>0, \quad \rho>0, r \in(0,1], \rho<r, \quad c \in(0,1) .
\end{gathered}
$$

Проведем анализ задачи на основе принципа максимума.

Составим функцию Понтрягина

$$
\begin{gathered}
H(\psi, x, u, v)=\psi r(c u+v) x+(1-u-v) x= \\
=H_{u}(\psi, x) u+H_{v}(\psi, x) v+x
\end{gathered}
$$

и сопряженное уравнение

$$
\dot{\psi}=\rho \psi-H_{x}=(1-\psi r c) u+(1-\psi r) v+\rho \psi-1
$$


с условием $\psi(T)=0$. формуле

Максимизирующее управления в поставленной задаче можно записать по

$$
\begin{aligned}
& u^{*}(\psi)=\left\{\begin{array}{ll}
0, & \psi r c-1 \leq 0 \\
1, & \psi r c-1>0
\end{array} ;\right. \\
& v^{*}(\psi)= \begin{cases}0, & \psi r-1 \leq 0 \\
1, & \psi r-1>0\end{cases}
\end{aligned}
$$

Рассмотрим задачу Коши для сопряженного уравнения

$$
\dot{\psi}=\psi\left(\rho-r\left(c u^{*}+v^{*}\right)\right)+\left(u^{*}+v^{*}-1\right), \quad \psi(T)=0
$$

с управлениями

$$
u^{*}=u^{*}(\psi), \quad v^{*}=v^{*}(\psi) .
$$

Пусть $\psi(t), t \in[0, T]$ - решение этого уравнения. Тогда управления $u^{*}(\psi(t)), v^{*}(\psi(t)), t \in[0, T]$ являются оптимальными в поставленной задаче.

Введем гладкие функции переключения, по которым будем отслеживать поведение управлений

$$
s_{1}(t)=\psi(t) r c-1, \quad s_{2}(t)=\psi(t) r-1 .
$$

Заметим, что при $r \in(0,1], c \in(0,1)$ возможны ситуации

$$
\begin{aligned}
& \text { - если } \psi(t)>0 \text {, то } \psi(t) r c-1<\psi(t) r-1 \Leftrightarrow s_{1}(t)<s_{2}(t) \text {; } \\
& \text { - если } \psi(t)<0 \text {, то } \psi(t) r c-1>\psi(t) r-1 \Leftrightarrow s_{1}(t)>s_{2}(t) .
\end{aligned}
$$

Начнем движение влево из терминальной точки $T$. Поскольку $\psi(T)=0$, то $s_{1}(T)=s_{2}(T)=-1$. Это означает, что $u^{*}(\psi(T))=0, v^{*}(\psi(T))=0$.

Следовательно, для $t \in(T-\varepsilon, T), \varepsilon>0$ имеем $s_{1}(t)<0, s_{2}(t)<0$. Отсюда вытекает, что $u^{*}(t)=0, v^{*}(t)=0$.

Подставляя в $\psi$-уравнение, получаем

$$
\dot{\psi}=\psi \rho-1, \quad \psi(T)=0 .
$$

Решением этого уравнения является функция

$$
\psi(t)=\frac{1}{\rho}\left(1-e^{\rho(t-T)}\right), \quad t \in(T-\varepsilon, T] .
$$

Продифференцируем полученное решение

$$
\dot{\psi}(t)=\frac{1}{\rho}(-\rho) e^{\rho(t-T)}=-e^{\rho(t-T)}<0, \quad t \in(T-\varepsilon, T] .
$$

Отсюда следует, что функция $\psi(t)$ - монотонно убывает на $(T-\varepsilon, T]$. Это означает, что $\psi(t)>0 \Rightarrow s_{1}(t)<s_{2}(t), t \in(T-\varepsilon, T]$. 
Таким образом, при движении по $t$ влево из $T$ первой обратится в нуль функция $s_{2}(t)$.

Решим уравнение $s_{2}(t)=0$, т.е.

$$
\frac{r}{\rho}\left(1-e^{\rho(t-T)}\right)-1=0 .
$$

В результате получена точка

$$
\theta_{1}=T+\frac{\ln \left(1-\frac{\rho}{r}\right)}{\rho}
$$

Убедимся в «правильности» найденной точки. Поскольку $1-\frac{\rho}{r} \in(0,1)$ (исходное условие $\rho<r)$, то $\ln \left(1-\frac{\rho}{r}\right)<0$.

Следовательно $\theta_{1}<T$.Отметим также, что $\psi\left(\theta_{1}\right)=\frac{1}{r}$.

Рассмотрим особый случай.

Пусть $\theta_{1} \leq 0$. Это означает, что $T \leq \frac{1}{\rho}\left|\ln \left(1-\frac{\rho}{r}\right)\right|$ (случай краткосрочного планирования). Тогда

$$
s_{1}(t)<0, \quad t \in[0, T], \quad s_{2}(t)<0, \quad t \in(0, T] .
$$

Следовательно, оптимальное управление в таком случае имеет вид

$$
u^{*}(t)=0, \quad v^{*}(t)=0, \quad t \in[0, T] .
$$

Пусть $\theta_{1}>0$. Тогда справедливо $s_{1}\left(\theta_{1}\right)<0, s_{2}\left(\theta_{1}\right)=0$.

Предположим, что $s_{2}(t)>0, t \in\left(\theta_{1}-\varepsilon, \theta_{1}\right)$, тогда

$$
u^{*}(t)=0, \quad v^{*}(t)=1, \quad t \in\left(\theta_{1}-\varepsilon, \theta_{1}\right) .
$$

Подставляя в $\psi$-уравнение, получаем

$$
\dot{\psi}=\psi(\rho-r), \quad \psi\left(\theta_{1}\right)=\frac{1}{r} .
$$

Решением этого уравнения является функция

$$
\psi(t)=\frac{1}{r} e^{(r-\rho)\left(\theta_{1}-t\right)}, \quad t \in\left(\theta_{1}-\varepsilon, \theta_{1}\right) .
$$

Заметим, что $\dot{\psi}(t)=\psi(t)(\rho-r)<0$. Отсюда следует, что $\psi(t)>0, \quad t<\theta_{1}$.

Проверим предположение $s_{2}(t)>0, \quad t \in\left(\theta_{1}-\varepsilon, \theta_{1}\right)$.

Согласно определению

$$
s_{2}(t)=\psi(t) r-1=e^{(r-\rho)\left(\theta_{1}-t\right)}-1 .
$$


Исследуем ее поведение. Функция $s(t)=e^{(r-\rho)\left(\theta_{1}-t\right)}$ монотонно убывает по $t \in\left[0, \theta_{1}\right]$, поскольку $\dot{s}(t)<0$, причем $s\left(\theta_{1}\right)=1$. Следовательно, $s(t)>1$ для всех $t \in\left[0, \theta_{1}\right)$. Отсюда вытекает, что $s_{2}(t)>0, t \in\left[0, \theta_{1}\right)$.

Поскольку $\psi(t)>0, \quad t \in\left[\theta_{1}-\varepsilon, \theta_{1}\right], \quad$ то $s_{1}(t)<s_{2}(t)$. Найдем решение уравнения $s_{1}(t)=0$

$$
c e^{(r-\rho)(T-t)}=1 .
$$

В результате получена точка

$$
\theta_{2}=\theta_{1}+\frac{\ln c}{r-\rho}
$$

Очевидно, что $\theta_{2}<\theta_{1}$. Отметим, что $s_{1}(t)<0, \quad t \in\left(\theta_{2}, \theta_{1}\right)$.

Выделим особый случай.

Пусть $\theta_{2} \leq 0$.Следовательно, $\theta_{1} \leq \frac{|\ln c|}{r-\rho}$. Тогда оптимальное управление имеет вид

$$
\begin{aligned}
& u^{*}(t)=0, \quad t \in[0, T], \\
& v^{*}(t)= \begin{cases}1, & t \in\left[0, \theta_{1}\right) \\
0, & t \in\left[\theta_{1}, T\right]\end{cases}
\end{aligned}
$$

Пусть $\theta_{2}>0$. Отметим, что $s_{1}\left(\theta_{2}\right)=0, \quad \psi\left(\theta_{2}\right)=\frac{1}{r c}$.

Предположим, что $s_{1}(t)>0, t \in\left(\theta_{2}-\varepsilon, \theta_{2}\right)$, тогда

$$
u^{*}(t)=1, \quad v^{*}(t)=1, \quad t \in\left(\theta_{2}-\varepsilon, \theta_{2}\right) \text {. }
$$

Решаем $\psi_{\text {-уравнение }}$

$$
\dot{\psi}=\psi(\rho-r(c+1))+1, \quad \psi\left(\theta_{2}\right)=\frac{1}{r c} .
$$

Решением этого уравнения является функция

$$
\psi(t)=e^{\left(\theta_{2}-t\right)(r(c+1)-\rho)}\left[\frac{1}{r c}-\frac{1}{r(c+1)-\rho}\right]+\frac{1}{r(c+1)-\rho} .
$$

Проверим предположение

$$
\begin{aligned}
& s_{1}(t)=\operatorname{cr} \psi(t)-1= \\
& =e^{\left(\theta_{2}-t\right)(r(c+1)-\rho)}\left[1-\frac{r c}{r(c+1)-\rho}\right]+\frac{r c}{r(c+1)-\rho}-1= \\
& =e^{\left(\theta_{2}-t\right)(r(c+1)-\rho)}\left[\frac{r-\rho}{r(c+1)-\rho}\right]+\frac{\rho-r}{r(c+1)-\rho}= \\
& =\frac{r-\rho}{r(c+1)-\rho}\left(e^{\left(\theta_{2}-t\right)(r(c+1)-\rho)}-1\right) \text {. }
\end{aligned}
$$

Так как $r-\rho>0$ и $r(c+1)-\rho>0$, то получим $\frac{r-\rho}{r(c+1)-\rho}>0$. 
Функция $e^{\left(\theta_{2}-t\right)(r(c+1)-\rho)}, t \in\left(0, \theta_{2}\right]$ в точке $\theta_{2}$ равна единице и монотонно убывает, поскольку ее производная меньше нуля. Следовательно, $e^{\left(\theta_{2}-t\right)(r(c+1)-\rho)}>1$. Проверим знак функции $\psi(t), \quad t \in\left(0, \theta_{2}\right)$

$$
\begin{gathered}
\dot{\psi}(t)=e^{\left(\theta_{2}-t\right)(r(c+1)-\rho)}\left(\frac{\rho-r}{r c}\right)<0, \\
\psi\left(\theta_{2}\right)=\frac{1}{r c}>0 .
\end{gathered}
$$

Следовательно, $\psi(t)>0, \quad t \in\left(0, \theta_{2}\right)$. Тогда $s_{2}(t)>s_{1}(t)>0, \quad t \in\left(0, \theta_{2}\right)$.

Таким образом, знаки функций переключения $s_{2}(t), s_{1}(t)$ на отрезке $[0, T]$ изменяются следующим образом:

1) функция $s_{1}(t)\left\{\begin{array}{ll}>0, & t \in\left(0, \theta_{2}\right) \\ <0, & t \in\left(\theta_{2}, T\right)\end{array}\right.$,

2) функция $s_{2}(t)\left\{\begin{array}{ll}>0, & t \in\left(0, \theta_{1}\right) \\ <0, & t \in\left(\theta_{1}, T\right)\end{array}\right.$.

Здесь $\theta_{1}=T+\frac{\ln \left(1-\frac{\rho}{r}\right)}{\rho}, \theta_{2}=\theta_{1}+\frac{\ln c}{r-\rho}$.

Итоговое условие $\theta_{2}>0$ приводит к неравенству для конечного времени $T>\frac{\left|\ln \left(1-\frac{\rho}{r}\right)\right|}{\rho}+\frac{|\ln c|}{r-\rho}$. Это случай долгосрочного планирования.

В этом случае оптимальное управление выражается по формулам

$$
\begin{aligned}
& u^{*}(t)=\left\{\begin{array}{ll}
1, & t \in\left[0, \theta_{2}\right) \\
0, & t \in\left[\theta_{2}, T\right]
\end{array},\right. \\
& v^{*}(t)= \begin{cases}1, & t \in\left[0, \theta_{1}\right) \\
0, & t \in\left[\theta_{1}, T\right]\end{cases}
\end{aligned}
$$
имеет вид:

Таким образом, содержательная интерпретация оптимального управления

- первый период $\left[0, \theta_{2}\right)$ - вся прибыль идет на инвестиции, берется кредит в размере прибыли;

- второй период $\left[\theta_{2}, \theta_{1}\right)$ - вся прибыль идет на инвестиции, кредит обнуляется;

- третий период $\left[\theta_{1}, T\right]$ - вся прибыль идет на потребление, кредит равен нулю.

$* * *$

1. Антипина Н. В. Линейные функции Ляпунова-Кротова и достаточные условия оптимальности в форме принципа максимума / Н. В. Антипина, В. А. Дыхта // Известия высших учебных заведений. Математика. — 2002. — № 12. - С. 11-22. 
2. Аргучинцев А. В. Оптимальное управление: нелокальные условия, вычислительные методы и вариационный принцип максимума / А. В. Аргучинцев, В. А. Дыхта, В. А. Срочко // Известия высших учебных заведений. Математика. - 2009. - № 1. - С. 3-43.

3. Габасов Р. Принцип максимума в теории оптимального управления / Р. Габасов, Ф. М. Кириллова. — М. :Либроком, 2011. - 272 с.

4. Кларк Ф. Оптимизация и негладкий анализ / Ф. Кларк. - М. : Наука, 1988. - 280 с.

5. Кротов В. Ф. Методы и задачи оптимального управления / В. Ф. Кротов, В. И. Гурман. - М. : Наука, 1973. - 446 с.

6. Математическая теория оптимальных процессов / Л. С. Понтрягин, В. Г. Болтянский, Р. В. Гамкрелидзе, Е. Ф. Мищенко. - М. : Физматлит, 1961. - 388 с.

7. Никольский M. C. О достаточности принципа максимума Понтрягина в некоторых оптимизационных задачах / М. С. Никольский // Вестник Московского университета. Сер. 15, Вычислительная математика и кибернетика. - 2005. - № 1. - С. 35-43.

8. Срочко В. А. Достаточные условия оптимальности в задачах управления на основе формул приращения функционалов / В. А. Срочко, В. Г. Антоник, Е. В. Аксенюшкина // Известия Иркутского государственного университета. Сер. Математика. — 2014. — Т. 8. - С. 125-140.

9. Срочко В. А. Достаточные условия оптимальности экстремальных управлений на основе формул приращения функционала / В. А. Срочко, В. Г. Антоник // Известия высших учебных заведений. Математика. - 2014. - № 8. - С. 96-102.

10. Срочко В. А. Итерационные методы решения задач оптимального управления / В. А. Срочко. - М. :Физматлит, 2000. - 160 с.

\section{Апекова А.O. \\ Оценка вероятности банкротства по методикам адаптированным к российской экономике}

Кузбасский государственный технический университет имени Т. Ф. Горбачева

(Россия, Кемерово)

doi:10.18411/spc-8-12-2017-03

idsp: 000001:spc-8-12-2017-03

Банкротство (несостоятельность) - признанная арбитражным судом или объявленная должником неспособность должника в полном объеме удовлетворить требования кредиторов по денежным обязательствам и (или) исполнить обязанность по уплате обязательных платежей [1].

Целью анализа вероятности банкротства является оперативное выявление близости к несостоятельности и применение необходимых мер в избежание данного состояния. Каждая из методик имеет право на существование, хоть и основываются на различных факторах. Для оценки вероятности банкротства АО «XXX» целесообразно рассмотреть следующие модели:

- методика Министерства финансов;

- четырехфакторная иркутская модель Беликова-Давыдовой;

- модель О.П. Зайцевой;

- модель Г.В. Савицкой.

Официальные методики созданы на основе отечественных нормативнозаконодательных документов, при помощи методического положения по оценке финансового состояния предприятий и установлению неудовлетворительной структуры баланса (№31 от 12 августа 1994 года) можно оценить вероятность банкротства, применяя систему из трех показателей:

1. Коэффициент текущей ликвидности (L тек. ликв.):

$\mathrm{L}$ тек. лик. $=\frac{\mathrm{A} 1+\mathrm{A} 2+\mathrm{A} 3}{\Pi 1+\Pi 2}$

$\mathrm{L}$ тек. лик. $2015=\frac{23714924}{7709013+20563414}=0,84$.

$\mathrm{L}$ тек. лик. $2016=\frac{26669857}{7884006+9337768}=1,55$.

2. Коэффициент обеспеченности собственными средствами (L об. соб. cp):

$\mathrm{L}$ об. соб. $\mathrm{cp.}=\frac{\Pi 4-\mathrm{A} 4}{\mathrm{~A} 1+\mathrm{A} 2+\mathrm{A} 3}$ 